\title{
Norois
}

Environnement, aménagement, société

$190 \mid 2004 / 1$

Varia

\section{Saint-Pierre et Miquelon, îles frontière}

\section{Christian Fleury}

\section{OpenEdition}

Journals

Édition électronique

URL : https://journals.openedition.org/norois/68

DOI : 10.4000/norois.68

ISBN : 978-2-7535-1538-3

ISSN : 1760-8546

\section{Éditeur}

Presses universitaires de Rennes

\section{Édition imprimée}

Date de publication : 1 janvier 2004

Pagination : $25-40$

ISBN : 978-2-86847-939-6

ISSN : 0029-182X

\section{Référence électronique}

Christian Fleury, « Saint-Pierre et Miquelon, îles frontière », Norois [En ligne], 190 | 2004/1, mis en ligne le 01 septembre 2008, consulté le 13 janvier 2022. URL : http://journals.openedition.org/norois/68 DOI : https://doi.org/10.4000/norois.68

Ce document a été généré automatiquement le 13 janvier 2022.

(C) Tous droits réservés 


\title{
Saint-Pierre et Miquelon, îles frontière
}

\author{
Christian Fleury
}

L'archipel français de Saint-Pierre et Miquelon est situé à une vingtaine de kilomètres de la côte sud de l'île canadienne de Terre-Neuve (fig 1). La frontière sépare deux territoires géographiquement et économiquement marginaux au sein de leurs États respectifs. L'archipel, collectivité territoriale au statut proche de celui d'un département, dispose pour environ 6300 habitants d'armatures institutionnelle et administrative réservées en métropole à une population en moyenne 100 fois plus importante. Il bénéficie d'importants transferts financiers et jouit d'un niveau de vie nettement supérieur à celui de voisins qui n'ont pas bénéficié du même soutien gouvernemental pour compenser les effets dévastateurs du moratoire canadien sur la pêche à la morue en 1992. Deux ans plus tard, le Canada et la France ont signé des accords dont l'objectif est de développer la coopération régionale et d'envisager les relations de voisinage selon une tonalité moins conflictuelle que par le passé. À SaintPierre et Miquelon, on semble disposé à utiliser les atouts que lui confèrent ses marginalités géographiques et institutionnelles pour diversifier une économie marquée par la dépendance. Géographiquement proche du Canada anglophone, l'archipel intensifie ses relations avec les communautés francophones du Canada atlantique. Il cherche également à développer des activités de dédouanement grâce d'une part à son statut en marge de l'Union européenne et d'autre part à sa situation à proximité des grands courants transatlantiques de marchandises. En outre, la ligne frontière marine tranche à la fois des stocks de poissons et de crustacés et des gisements d'hydrocarbures, éléments transfrontaliers non réductibles aux limites inter étatiques. Leur gestion nécessite donc des mécanismes complexes d'ajustement. Conçu selon une double approche, diachronique et synchronique, ce texte s'attache à décrire les interactions frontalières mises en œuvre à différents niveaux de l'échelle spatiale, du plus près de la ligne virtuelle elle-même, c'est-à-dire en mer, à celles géographiquement plus diffuses des relations culturelles. 
Figure 1 : La situation de Saint-Pierre et Miquelon

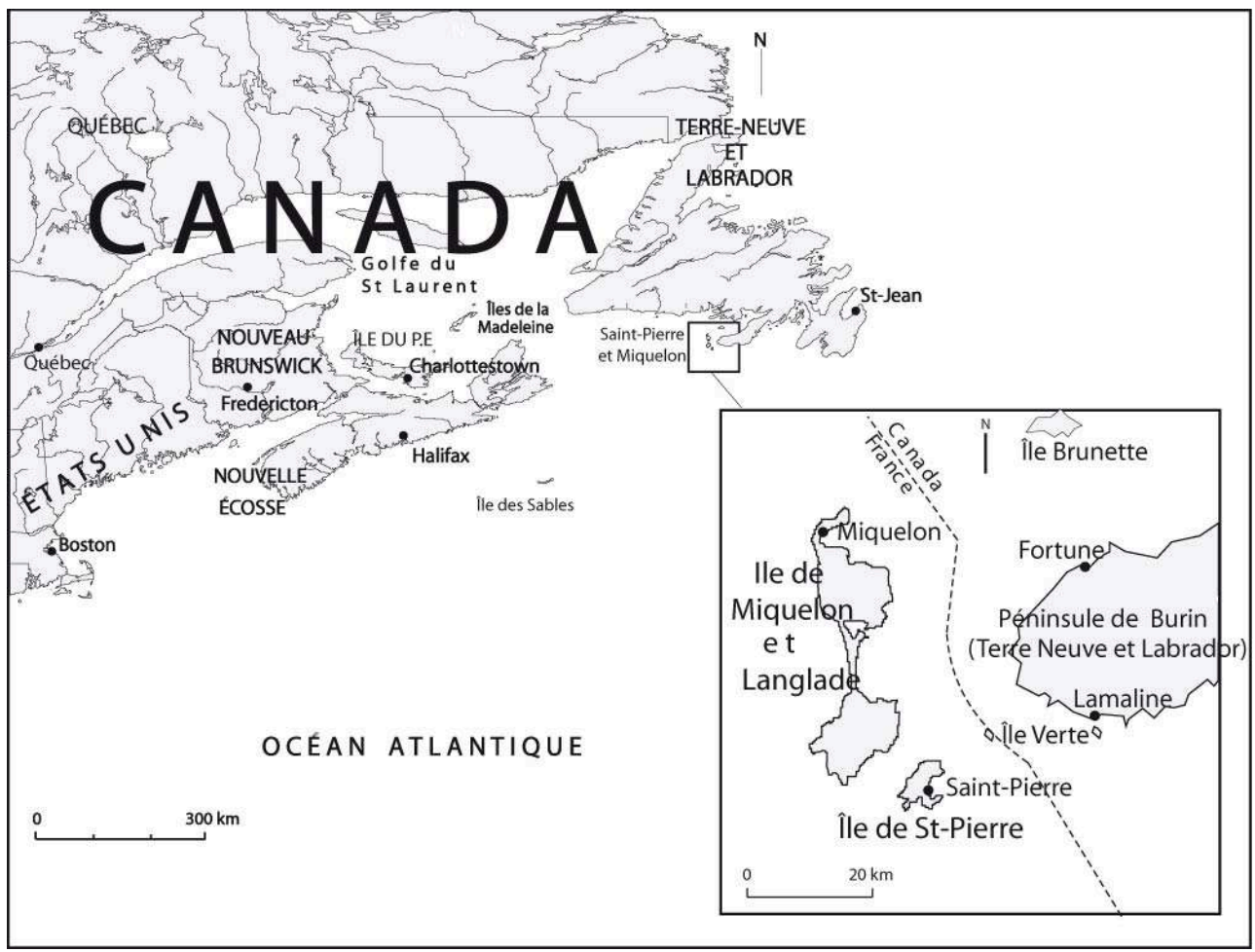

Fleury 2003

\section{Saint-Pierre et Miquelon, à proximité des bancs de Terre-Neuve et de la sphère anglaise}

Depuis sa prise de possession par Jacques Cartier au nom de François $1^{\text {er }}$ en 1536 et jusqu'en 1713, date de sa cession à l'Angleterre en vertu d'une des clauses du traité d'Utrecht (fig. 2), l'archipel est resté faiblement peuplé, de l'ordre de quelques dizaines de personnes. Le navigateur malouin y rencontre des pêcheurs venus de France. Mais le caractère saisonnier de la pêche à la morue qui abonde dans ses environs ne favorise alors pas un développement rapide d'une colonie. La rétrocession à la France en 1763 lors du traité de Paris en fait le seul établissement français en Amérique du Nord, tout entier dévolu à une fonction de point d'appui à la grande pêche métropolitaine, ellemême considérée comme un instrument de formation irremplaçable pour les futures recrues de la Marine Royale. Mais dès cette date, sa situation à proximité de leur patrie désormais sous domination anglaise, conduit un certain nombre de familles d'origine acadienne à considérer l'archipel, et en premier lieu Miquelon, comme un refuge où l'installation n'exclurait pas un éventuel retour sur le continent américain. En effet, la population francophone est expulsée au fur et à mesure de la radicalisation de la politique anglaise en Acadie, territoire discontinu comprenant des établissements de population d'origine française dans les régions correspondant aux provinces actuelles de Nouvelle-Écosse, de l'île du Prince-Édouard et du Nouveau-Brunswick. Depuis 1713, la France ne possède plus en Amérique du nord que l'île Saint-Jean (aujourd'hui île du Prince-Édouard) et l'île Royale (aujourd'hui île du Cap-Breton) sur laquelle a été édifiée la forteresse de Louisbourg. Les populations francophones restées dans les secteurs 
désormais sous domination anglaise sont de plus en plus menacées jusqu'aux déportations du Grand Dérangement en 1755. Celui-ci a pour conséquence de pousser un certain nombre d'Acadiens à mener une vie errante des deux rives de l'Atlantique pendant plus de cinquante ans, dans l'espoir entêté d'un hypothétique retour en Acadie. Saint-Pierre et Miquelon représente désormais un point d'ancrage pour les plus obstinés, que la volonté royale de ne pas favoriser une installation étrangère à la filière halieutique ne parvient pas à décourager. Jusqu'en 1815, la population vit une alternance de démarrages économiques et de déracinements puisqu'elle est déportée par les Anglais en 1778 et 1793 après avoir été renvoyée en métropole en 1767 sur ordre de Choiseul qui autorise cependant son retour un an plus tard.

Figure 2 : Changements de souveraineté de Saint-Pierre et Miquelon entre 1713 et 1815

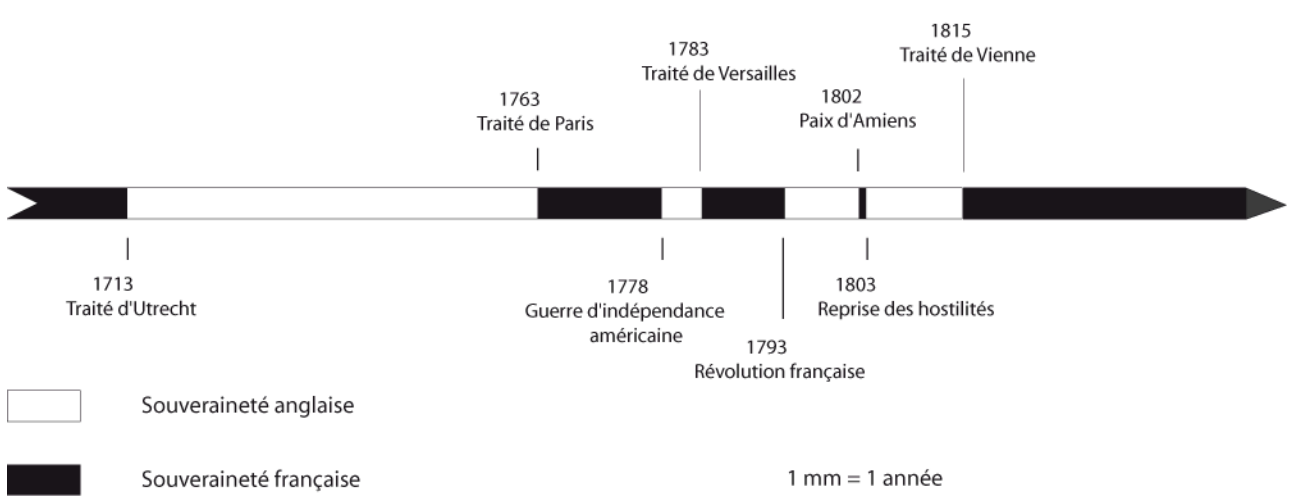

3 Le traité de Vienne en 1815 marque la fin définitive des conflits armés entre la France et l'Angleterre et mis à part quelques velléités sans suite d'annexions par le Canada ou les États-Unis, notamment dans le contexte des deux guerres mondiales (Guyotjeannin, 1986), la souveraineté française n'est plus remise en cause. Le choix de la France, qui préfère, selon le marché proposé par l'Angleterre, garder Saint-Pierre et Miquelon plutôt que l'île de France, aujourd'hui île Maurice, confirme sa volonté de maintenir une base de pêche dans le secteur avec le même objectif sous-jacent de former une grande quantité d'hommes aux dures conditions de la vie en mer. Pas moins de 12000 navires et 450000 hommes auraient ainsi constitué les effectifs de l'armement terreneuvier français tout au long du XvIII ${ }^{e}$ siècle (Brière, 1990). Saint-Pierre développe au cours du XIX siècle une activité de station service à proximité des bancs de TerreNeuve, zone de hauts fonds et de confluence des eaux douces du fleuve Saint-Laurent et de courants marins chaud, le Gulf Stream, et froid, le Labrador. Ces conditions en font un secteur particulièrement riche en morues attirées par l'abondance de microorganismes constituant le début de la chaîne alimentaire. L'archipel est alors essentiellement tourné vers le large et le lointain, servant de plate-forme d'avitaillement, de débarque et de transit pour les navires métropolitains ou issus d'autres pays pratiquant la grande pêche. Cette fonction dure, avec des hauts et des bas, jusqu'au moratoire canadien de 1992, décision qui a également comme conséquence de tarir l'approvisionnement de l'usine de transformation du poisson qui emploie alors environ 300 personnes à Saint-Pierre et y constitue la seule activité industrielle. La diversité du peuplement est une conséquence de cette inclusion de l'archipel dans la filière halieutique transatlantique. En effet, à partir du substrat acadien, différents apports contredisent le cliché l'assimilant parfois à un isolat démographique (tableau 1). 
Tableau 1 : Répartition du lieu de naissance des personnes ayant contracté un mariage à SaintPierre et Miquelon entre 1816 et 1889

\begin{tabular}{|l|c|c|}
\hline \multicolumn{1}{|c|}{ Lieu de naissance } & Nombre & Pourcentage \\
\hline France dont : & 2872 & $\mathbf{8 9 , 3 3}$ \\
SAINT-PIERRE ET MIQUELON & 1437 & 44,69 \\
MÉTROPOLE & 1435 & 44,63 \\
$\quad$ dont (départements) : & & \\
Manche & 561 & 17,44 \\
Basse-Pyrénées & 233 & 7,24 \\
Ille-et-Vilaine & 222 & 6,90 \\
Côtes-d'Armor & 133 & 4,13 \\
Autres & 286 & 8,89 \\
\hline Étranger & 343 & $\mathbf{1 0 , 6 6}$ \\
TERRE-NEUVE & 189 & 5,87 \\
Autres & 154 & 4,66 \\
\hline
\end{tabular}

(source : Girardin, 1990)

4 L'origine des contractants français se répartit presque également entre Saint-Pierre et Miquelon et la métropole. Certains pêcheurs métropolitains, essentiellement d'origine normande, bretonne ou basque, viennent d'abord seuls faire la saison de pêche puis font venir leur famille et s'installent à l'année. D'autres hivernent à Saint-Pierre pour éviter le coût d'un aller et retour, se marient sur place, parfois avec une TerreNeuvienne. Vers 1850, les conserveries basques se développent et amènent leurs employés. Les fonctionnaires métropolitains constituent progressivement une autre composante de la population.

5 L'assimilation des personnes originaires de Terre-Neuve se fait en douceur et sans conséquence linguistique. Elle concerne essentiellement des femmes, ce qui explique le peu de traces dans l'anthroponymie locale des noms à consonance anglo-saxonne, alors que Saint-Pierre et Miquelon se situe à 20 kilomètres du Canada anglophone. Sur les 189 personnes nées à Terre-Neuve recensées par Girardin (1990), on compte seulement 18 hommes. Deux facteurs concordants incitent ces femmes à venir à Saint-Pierre. Il s'agit d'une part de la misère à laquelle est confrontée la population essentiellement d'origine irlandaise des petites communautés littorales du sud de Terre-Neuve et d'autre part du besoin d'emplois notamment au cours des périodes de prospérité dont l'exemple le plus significatif est au $\mathrm{xx}^{\mathrm{e}}$ siècle la période de la Prohibition après la première guerre mondiale. La fin du siècle précédent est également une période de dynamisme économique qui se traduit par l'arrivée de jeunes Terre-Neuviennes destinées aux emplois de domestiques. Ainsi au cours de la période 1880-1889, correspondant d'une part à la dernière décennie recensée par Girardin et d'autre part 
au début d'un épisode économique dynamique pour l'archipel, 75 mariages concernent des Canadiennes venues de la grande île voisine. Les Mi'kmaks, peuple amérindien allié traditionnel des Français lors des conflits avec les Anglais, représentent un autre apport démographique jusqu'à maintenant peu mis en évidence ${ }^{1}$.

6 Ce brassage constitue un élément de fierté dans le discours d'un certain nombre d'habitants. Telle personne, au patronyme d'origine normande, a du sang amérindien par sa grand-mère. La mère de telle autre, portant un nom basque par son mariage, est native de Terre-Neuve. Sans que cela ait été démontré par une enquête, des propos concordants, font état d'une estimation de l'ordre de $50 \%$ d'habitants ayant une ascendance terre-neuvienne. Mais Saint-Pierre et Miquelon entretient avec son voisinage canadien des relations marquées par le déséquilibre. L'archipel bénéficie de l'ouverture apportée par des activités intégrées dans un vaste mouvement pendulaire transatlantique alors que sur le littoral terre-neuvien, les communautés de pêcheurs sont, en plus de la dureté des conditions climatiques commune à tous dans la région, confrontées le plus souvent à d'importantes difficultés d'ordre économique. Ces relations sont ambivalentes dans la mesure où elles regroupent à la fois des liens matrimoniaux, des échanges économiques, y compris sous forme de contrebande, et des rapports tendus avec les autorités terre-neuviennes qui ont du mal à accepter la présence d'un « corps étranger » à proximité de leurs côtes et qui reprochent d'ailleurs au gouvernement britannique, qui restera son autorité tutélaire jusqu'à l'intégration de Terre-Neuve dans la confédération canadienne en 1949, son peu d'empressement à défendre ses intérêts. La promulgation du Bait Bill en 1887 illustre le conflit d'intérêt entre les autorités et une partie de la population qui s'accommode du voisinage de l'archipel en profitant de son dynamisme. Cette loi interdit la vente de boette, constituée de poissons destinés à servir d'appâts pour la morue, aux pêcheurs de SaintPierre et Miquelon afin de ne pas contribuer ainsi à leur effort de pêche. Cette marque déclarée d'hostilité n'est pas bien acceptée par les pêcheurs terre-neuviens qui vivent de ce trafic. Il y a là en matière de relations frontalières plusieurs niveaux de lecture : l'exercice par ses riverains de la frontière au quotidien qui joue notamment sur les différentiels économiques, le jeu conflictuel des autorités locales poussées par des opinions publiques globalement hostiles à la présence étrangère voisine et les relations de niveau supérieur entre les puissances tutélaires qui agissent en fonction d'une combinaison d'intérêts à l'échelon planétaire. Ainsi, les Terre-Neuviens manifestent-ils leur colère et leur incompréhension lorsque le Royaume Uni consent au maintien de la souveraineté de la France vaincue de 1815 sur Saint-Pierre et Miquelon. De l'autre côté, la renonciation par la France en 1904 aux droits de pêche exclusifs sur une partie du littoral terre-neuvien, le French Shore, est très mal reçue sur l'archipel.

7 La propension à se sentir mal défendu par la métropole est un élément diachronique très présent dans le dispositif mental de la population. Elle s'associe à un sentiment de méfiance vis-à-vis du voisin canadien. Ce double ressentiment est notamment alimenté par les avatars rencontrés en matière d'appropriation de l'espace marin. En effet, les questions de partage de la mer se sont substituées à celles touchant à la souveraineté des terres, réglées depuis presque deux siècles. 


\section{Les conflits frontaliers marins}

8 Les velléités croissantes de territorialisation de l'espace marin par les États constituent un phénomène géopolitique majeur de la deuxième partie du xx siècle. À partir de 1945 et de la déclaration unilatérale du président américain Truman, on assiste à une course à l'annexion par les États d'une part de plus en plus importante de la mer, d'abord à travers des revendications sur le plateau continental, puis ensuite sur les eaux ellesmêmes. Ceci ne va pas sans entrer en collision avec les droits historiques en matière de pêche. La territorialisation de l'espace marin, définie en 1982 à Montego Bay selon un modèle théorique, entraîne la multiplication de conflits souvent difficiles à régler. La combinaison île-frontière, telle qu'on la retrouve dans le secteur de Saint-Pierre et Miquelon, cumule les conditions propices à leur émergence. Jusqu'à la seconde guerre mondiale, la mer peut y être considérée comme largement ouverte dans la mesure où il existe peu d'entraves à la pratique halieutique autres qu'économiques ou liées à une carence de la ressource. Dans la nomenclature fournie des conflits d'appropriation de l'espace marin, la frontière maritime franco-canadienne représente un cas parmi d'autres. Il convient cependant d'évoquer la situation particulière occupée par le Canada, au niveau international à propos de ces questions. Premier ou deuxième du monde, au niveau de la Russie, selon des estimations divergentes par la superficie de son plateau continental et la longueur de ses côtes, il fait partie des pays les plus prospères de la planète. Cependant, il fait figure de «nain géopolitique » dans la mesure où sa posture internationale est perçue comme étroitement dépendante de celle des États-Unis, sans oublier son appartenance, même si elle est symbolique, à la Couronne britannique. Or, il trouve dans les dynamiques territoriales concernant la mer qui se mettent progressivement en place à partir des années 1950 de quoi asseoir son audience internationale. Le Canada, souvent en opposition avec ses alliés qui considèrent que, vu leur suprématie technologique et militaire, ils ont plus à perdre qu'à gagner en acceptant l'annexion par tous les États côtiers de leur mer adjacente, trouve des partenaires de poids dans les pays alors dits "du tiers monde» et notamment avec les plus influents et les plus impliqués dans la dynamique de la conférence de Bandung en 1955 comme le Mexique, le Brésil ou l'Indonésie. Cet activisme sur la scène mondiale a des implications locales.

9 À partir de la fin des années 1970 et de l'instauration des Zones Économiques Exclusives de 200 milles, le Canada cherche à réguler l'intense activité halieutique sur les bancs. On ne doit également pas perdre de vue le fait que d'autres enjeux concomitants et, au propre et au figuré, sous-jacents, se font jour dans la région. En effet, depuis les années 1960 les prémices d'un conflit entre la France et le Canada se mettent en place à propos de la forte présomption de présence d'importants gisements d'hydrocarbures dans la région. À cet égard, on peut voir une illustration du fait que les enjeux marins s'enfoncent. De maritime, donc concernant la surface et la circulation des navires à des fins stratégiques, les conflits marins se sont progressivement étendus à la colonne d'eau à propos des questions halieutiques avant de concerner également le sol et le sous-sol y compris, en raison des progrès technologiques, à de grandes profondeurs. On retrouve ce processus dans le secteur marin autour de Saint-Pierre et Miquelon. Marqué jusqu'en 1815 par des conflits où les entraves à la circulation des navires s'inscrivaient davantage dans un projet d'appropriation terrestre que maritime, il a ensuite été le théâtre de nombreuses querelles de voisinage à propos des pêcheries de 
morues et de crustacés. Ces conflits se sont progressivement cristallisés lorsque les droits historiques de pêche se sont trouvés en collision avec l'établissement de limites territoriales que l'on qualifie souvent de "maritime » alors qu'en réalité, elles sont «marines » et "sous-marines ", c'est-à-dire concernant la mer dans toute sa verticalité. Les frontières qui s'inscrivent sur le bleu des cartes posent ainsi un double problème, spatial puisque elles tranchent des éléments dynamiques et animaux cohortes de poissons ou de crustacés - ou statiques et minéraux - gisements de granulats ou d'hydrocarbures - et temporel, puisque leur mise en place intervient après plusieurs siècles de pratiques halieutiques qui les ignoraient.

Le Canada est un des États en pointe dans la revendication, finalisée à Montego Bay, de la création d'une Zone Économique Exclusive (ZEE) d'une largeur de 188 milles (soit jusqu'à une distance totale de 200 milles des lignes de base en incluant les 12 milles de la mer territoriale) où l'État riverain dispose de droits souverains sur les ressources biologiques et minérales (fig. 3). Décrétée unilatéralement par un certain nombre d'États dont le Canada dès 1977, son établissement déclenche un grand nombre de litiges dans la mesure où le modèle théorique est, en raison des circonstances particulières et notamment la configuration des côtes, souvent inopérant. La présence d'îles étrangères a généralement entrainé des désaccords dont la résolution est, dans le cas le plus fréquent d'absence d'entente bilatérale, confiée à des arbitrages internationaux. Le Canada a pu montrer sa pugnacité avec les trois pays avec lesquels il possède des frontières marines. La question de la frontière dans le golfe du Maine entre le Canada et les États-Unis a fait l'objet d'une longue et âpre négociation en définitive tranchée par la Cour internationale de justice en 1984, plutôt à l'avantage du Canada (Labrecque, 1998). Du côté du Pacifique, les frontières marines entre les deux pays sont toujours virtuelles. Entre le Canada et le Danemark, représentant les intérêts du Groenland, un accord partiel a été signé. Enfin, les conditions du règlement du litige entre la France et le Canada soulèvent toujours dix ans plus tard l'indignation des habitants de Saint-Pierre et Miquelon. 
Figure 3 : Saint-Pierre et Miquelon et sa ZEE

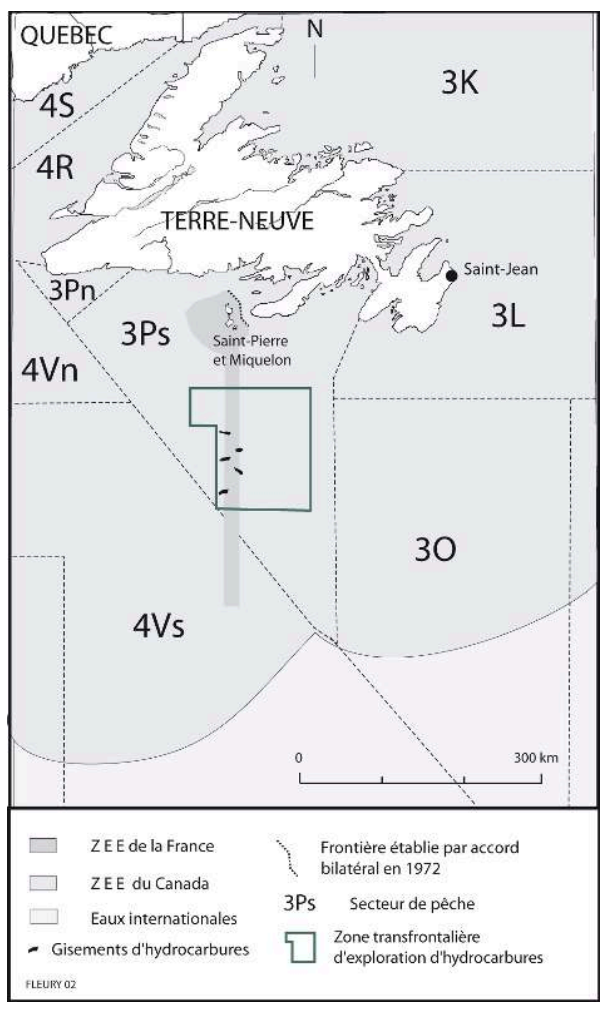

11 En effet, le 10 juin 1992, le tribunal arbitral de New York rend son verdict en ce qui concerne la délimitation de la ZEE française autour de Saint-Pierre et Miquelon. Le jugement y est très mal reçu puisque le secteur attribué par l'instance internationale occupe moins du quart de la superficie réclamée par la France. Sa forme constitue un autre sujet de mécontentement. Appelé depuis lors le «tuyau » par la population, il fait bien les 188 milles de long prévus par la Convention de Montego Bay mais uniquement vers le sud sur une largeur de 10,5 milles marins, c'est-à-dire strictement la projection maximale est-ouest des côtes de l'archipel.

En outre, le fait, qu'en raison d'effets induits par l'île néo-écossaise des Sables au sudouest et par la péninsule terre-neuvienne d'Avalon au nord-est, la ZEE française ne débouche pas sur la haute mer constitue une caractéristique très mal ressentie même si les effets de cet enclavement sont essentiellement symboliques. La plaidoirie canadienne défendant la position selon laquelle Saint-Pierre et Miquelon doit être considéré comme une enclave trouve ici une application maximale puisque les exemples de ZEE enclavées sont à notre connaissance extrêmement rares². Il convient d'ailleurs de relever la grande différence de moyens mis en œuvre par chacune des parties pour faire valoir son point de vue. Pendant plusieurs années, le Canada a mobilisé sur cette affaire ses spécialistes du droit international et du droit de la mer au service d'avocats qui ont plaidé avec efficacité pour la reconnaissance du concept flou de « circonstances spéciales ». Face à cette machine juridique bien organisée, la France a opposé l'argument de l'équidistance stricte, défendue par une délégation mise, selon des observateurs locaux proches du dossier, dans ces conditions matérielles incompatibles avec la volonté évidemment affichée par les autorités de défendre au mieux les intérêts français. L'épilogue très défavorable à la France n'est en définitive guère surprenant. Il s'ajoute la même année à une autre décision lourde de 
conséquences pour l'archipel, à savoir le moratoire sur la pêche à la morue décidé par le Canada à l'intérieur de sa ZEE, c'est-à-dire sur la quasi totalité des bancs.

13 La situation des stocks de morue est préoccupante depuis les années 1980. Devant la dégradation d'une situation qu'il a contribué à aggraver en vendant des licences par centaines à des navires, soviétiques, polonais et espagnols pour les plus gros armements, aux procédés destructeurs, le Canada décide un moratoire sur la morue. Pour Saint-Pierre et Miquelon, cela représente un coup fatal non seulement à la pêche locale mais également et peut-être surtout aux deux fonctions de la filière halieutique qui la font vivre en amont et en aval, l'avitaillement et la transformation du poisson. En 1969, 1104 escales de navires étrangers - dont 619 espagnols - avaient été comptabilisées (Cermakian et al., 1970) contre seulement 43 en 2001 (IEDOM, 2002).

14 Le ressentiment contre d'une part le voisin canadien et d'autre part la métropole est alors à son comble. C'est dans ce contexte qu'interviennent deux ans plus tard les accords de 1994, signés au plus haut niveau, celui des premiers ministres. Leur objectif est de sortir d'une logique d'affrontement et fixer le cadre de relations apaisées et constructives. Une Commission Mixte de Coopération Régionale est instaurée. Elle s'est réunit chaque année sauf en 1998 où des tensions sur la pêche n'ont pas permis leur tenue. Sa fonction consiste à favoriser et à assurer le suivi de dossiers de coopération entre Saint-Pierre et Miquelon et le Canada. Les accords de 1994 établissent les bases de nouvelles relations selon lesquelles, les questions porteuses de conflits ayant été de fait évacuées par la décision canadienne, les conditions sont paradoxalement réunies pour le développement d'une coopération régionale basée sur le principe du "gagnantgagnant » expression directement reprise de l'Américain win win et qui revient souvent dans les propos des personnes impliquées. Cette volonté, tournant le dos à une logique d'affrontement, est parfois considérée sur l'archipel comme un "os à ronger " pour faire passer la «pilule amère » des décisions de 1992. D'autres voix, plus positives, considèrent que les accords de 1994 constituent une opportunité de s'affranchir de la dépendance et de jouer les diversifications qui s'imposent d'une part à l'intérieur de la filière halieutique et d'autre part vers d'autres activités.

Les dispositions prises en 1997 pour 10 ans établissent les conditions d'une reprise très partielle de la pêche à la morue. Dans le secteur du 3Ps (fig. 3), les Français se voient attribuer $15,6 \%$ des quotas établis en commun chaque année. Mais $70 \%$ de ces prises doivent être effectuées par un navire et un équipage canadien. Les $30 \%$ restants sont dévolus à la petite pêche de l'archipel. Il y a là de quoi simplement maintenir quelques dizaines d'emplois à Interpêche mais en aucun cas permettre un véritable redémarrage d'une activité halieutique intégrée sur l'archipel. D'autres quotas, consentis par le Canada à Saint-Pierre et Miquelon hors 3Ps au sud-ouest de Terre-Neuve s'avèrent impossibles à pêcher en raison de l'inadéquation des clauses de l'accord avec la structure de la flottille locale. Les propositions d'échanges de quotas avec les Canadiens n'ont pas été jusqu'à maintenant prises en compte. Confrontés à ces difficultés et à la baisse drastique des apports, la filière pêche de l'archipel s'est lancée dans la diversification, en s'ouvrant à des espèces naguère délaissées comme le crabe des neiges, le bulot, le pétoncle. Les résultats sont mitigés comme en témoignent la disparition du gisement de pétoncles, victime de la prédation de l'étoile de mer, et, pour l'année 2002, la migration vers le sud, donc hors d'atteinte des pêcheurs de l'archipel, de la cohorte de crabe des neiges. Cette dernière pêcherie illustre le décalage entre les bonnes volontés affichées et le difficile exercice de la cohabitation. Selon des 
sources françaises les pêcheurs de Saint-Pierre et Miquelon n'auraient pas été prévenus en mai 2002 par les autorités canadiennes de la date de reprise de la pêche, et ceci contrairement aux dispositions prévues par l'accord de 1997.

En outre, la présence de gisements transfrontaliers d'hydrocarbures agite d'une façon plus discrète les relations entre la France et le Canada depuis plusieurs décennies. La ZEE de Saint-Pierre et Miquelon, aussi étroite qu'elle soit, tranche d'importantes réserves (fig. 3). L'ensemble des règles destinées à définir de façon extrêmement précise les droits et devoirs de chacune des parties dans les domaines de l'exploration, de l'exploitation, des redevances, de l'emploi et de l'avitaillement sont regroupées dans un traité dit d'unitisation actuellement en cours de négociation entre les deux pays. Les développements les plus récents révèlent d'importantes divergences de point de vue. En mai 2003, les discussions sont dans une impasse en raison d'un blocage canadien sur la possibilité pour Saint-Pierre et Miquelon d'armer des navires destinés au ravitaillement des plates-formes canadiennes.

17 Les difficultés de la filière pêche ont conduit les milieux économiques et politiques à miser sur la coopération régionale dans un souci de diversification cette fois-ci externe à la pêche. L'archipel tente ainsi de jouer de ses différentes qualités de marges, à la fois française, francophone et européenne en Amérique du Nord et d'étendre les spectres économique, culturel, spatial de ses pratiques frontalières.

\section{Saint-Pierre et Miquelon et la péninsule de Burin}

Lorsque l'on considère la proximité entre l'archipel français de Saint-Pierre et Miquelon et la péninsule canadienne de Burin sur l'île de Terre-Neuve, on constate qu'elle concerne deux régions à la fois périphériques et marginales dans leur contexte national respectif.

L'une et l'autre présentent bien une situation d'éloignement géographique par rapport au centre de leur entité nationale respective. Saint-Pierre et Miquelon dans la nomenclature des Départements d'Outre-Mer (DOM) et des Pays et Territoires d'OutreMer (PTOM) appartenant à la France ${ }^{3}$ présente la particularité d'être le plus proche de la métropole - à peine 4000 kilomètres - et le territoire dont l'accès est parmi les plus onéreux, du même ordre que pour la Nouvelle-Calédonie ou la Polynésie, 5 fois plus éloignées. Le vol aller et retour métropole/Saint-Pierre via Montréal coûte au minimum $1000 €$ en basse saison et son prix peut grimper jusqu'à environ $1500 €$ en période estivale. Des solutions à peine moins coûteuses et encore plus pénalisantes en terme de temps existent par Saint-Jean de Terre-Neuve ou Halifax via Londres. La continuité territoriale ne s'applique d'ailleurs pas à Saint-Pierre et Miquelon puisque son accès passe obligatoirement par un changement de vol au Canada, pays qui ne reconnaît pas la notion de transit. Un passager français est donc tenu de présenter un passeport au personnel de l'immigration canadienne avant de reprendre l'avion pour Saint-Pierre.

La péninsule de Burin peut également être considérée comme périphérique dans le contexte canadien. La route transcanadienne finit - ou commence - son parcours de 9000 kilomètres au bord de l'Atlantique à Saint-Jean de Terre-Neuve. À partir d'une jonction située à environ 150 kilomètres à l'ouest de Saint-Jean, 200 kilomètres restent à parcourir vers le sud-ouest pour se rendre à Fortune, petite ville portuaire d'environ 2000 habitants d'où un traversier conduit à Saint-Pierre en une heure, de mai à septembre. Certaines petites communautés de pêcheurs de la côte sud de Terre-Neuve, 
dans la région de la baie de l'Hermitage par exemple, ne sont pas desservies par un réseau routier asphalté dont elles sont parfois éloignées de plusieurs dizaines de kilomètres.

21 La périphérie, attribut géographique, se double dans le cas de Saint-Pierre et Miquelon comme dans celui de la péninsule de Burin d'une marginalité économique, augmentée pour l'archipel français de particularités institutionnelles. Cependant il s'agit de marginalités économiques opposées de part et d'autre de la ligne frontière dans la mesure où les deux espaces considérés présentent des structures économiques et des profils statistiques très divergents.

Saint-Pierre et Miquelon, après avoir changé trois fois de statut depuis 1945 est depuis 1985 une Collectivité territoriale au sein de la République Française. Proche de celui d'un département, il en diffère cependant sur certains points importants. Le conseil général, organe exécutif, dispose des autonomies fiscale et douanière. Par ailleurs, il possède un rôle consultatif sur les questions régionales, donc frontalières avec le Canada. Les 6316 habitants de Saint-Pierre et Miquelon (source: INSEE, 1999) bénéficient d'armatures administrative et institutionnelle à peu près équivalentes à celles d'un département métropolitain, en moyenne presque 100 fois plus peuplé (tableau 2). La structure de l'emploi rend compte de cette disproportion puisque, pour l'année 2000 , on a recensé 825 emplois de fonctionnaires ou assimilés qui bénéficient d'une bonification salariale de 40 à $70 \%$ par rapport à la métropole. Parmi les emplois atypiques pour une communauté de petite taille mais induits par la situation insulaire et périphérique de l'archipel, on peut citer les exemples du service de l'Aviation Civile qui emploie 37 personnes et l'antenne de Radio France Outre-mer (RFO) qui compte une soixantaine de collaborateurs.

Économiquement et symboliquement touché par le moratoire canadien sur la pêche à la morue sur les bancs de Terre-Neuve en 1992 très partiellement levé en 1997, l'archipel vit surtout des transferts salariaux de la fonction publique et a vu l'activité de sa filière halieutique maintenue grâce aux subventions gouvernementales. Ces sources de revenus confèrent aux habitants de Saint-Pierre et Miquelon une position globalement enviable dans un proche environnement régional très déprimé et ceci malgré un taux de couverture des importations par les exportations qui, depuis la chute de l'activité de transformation de la morue, se situe bon an mal an autour de $15 \%$.

Tableau 2 : Évolution 1991-2001 du nombre d'habitants et taux de chômage dans la péninsule de Burin et à Saint-Pierre et Miquelon

\begin{tabular}{|l|c|c|c|c|}
\hline & Population en 1991 & Population en 2001 & Variation en \% & Taux de chômage \\
\hline Burin & 2940 & 2470 & $-15,99$ & 29,9 \\
Fortune & 2177 & 1615 & $-25,82$ & 58,3 \\
Grand Bank & 3528 & 2841 & $-19,47$ & 25,8 \\
Lamaline & 482 & 348 & $-28,22$ & 57,6 \\
Lawn & 1005 & 779 & $-22,49$ & 50 \\
Marystown & 6739 & 5908 & $-12,33$ & 33,9 \\
St Lawrence & 1743 & 1558 & $-10,81$ & 39,1 \\
Saint-Pierre et Miquelon & 6277 & 6316 & $+0,62$ & 9,5 \\
& (en 1990) & (en 1999) & & (moyenne 2000) \\
\hline
\end{tabular}

(sources : Statistiques Canada 2001 et IEDOM) 
24 Terre-Neuve ont été touchées au même titre que Saint-Pierre et Miquelon par le moratoire sur la morue décidé en 1992 par leur gouvernement fédéral. Mais celui-ci n’a pas appliqué les mêmes dispositions aux vertus apaisantes que son homologue français. La différence de stratégie gouvernementale de part et d'autre de la frontière concernant des communautés proches touchées par la même décision est patente et illustre le propos de Claude Raffestin (1983) qui estime que la frontière "sépare deux systèmes d'intention et d'action». Les gouvernements français ont compensé une implication jugée timorée sur l'archipel dans les grands dossiers touchant aux questions territoriales marines par une politique visant à étouffer les mécontentements sous de substantiels apports financiers. Ceux-ci permettent à l'archipel de présenter des indicateurs économiques et démographiques qui ne laissent pas transparaître les effets négatifs de la crise de la pêche.

La gestion de la crise de la morue par les gouvernements canadiens, fédéral et provincial, est fondamentalement différente. Les théories néo-conservatrices estimant qu'il faut laisser le marché restaurer l'équilibre économique quitte à provoquer le déplacement de population ont été largement appliquées du côté canadien de la frontière. Cette conception nord américaine privilégie une vision libérale des rapports entre l'homme et ses environnements économique et spatial. Donald J. Savoie (2000) définit une hiérarchie dans les communautés du Canada atlantique susceptibles de trouver des axes de diversification économiques. Il distingue ainsi trois catégories selon leur degré de dépendance à l'activité historique désormais abandonnée à savoir «les communautés qui disposent d'une solide infrastructure administrative et présentent une certaine diversité économique ", ensuite celles " qui offrent quelques perspectives de diversification économique mais qui demeurent pauvres en matière d'infrastructures et de soutien administratif » et enfin «les communautés qui ont été totalement dépendantes des pêches, qui comptent peu de chefs d'entreprise et dont les ressources administratives sont trop rares pour qu'elles puissent concevoir, planifier, organiser et entreprendre d'autres activités économiques». Cette classification constitue un guide de nature à déterminer quelles sont les communautés dont la survie est possible et où l'injection d'argent public est souhaitable. Le degré de crise est un critère moins décisif que le potentiel de rebondissement. Selon cette approche, les communautés littorales et les petites villes du sud de Terre-Neuve sont peu éligibles aux stratégies de diversification. Les habitants sont alors fortement incités à aller chercher du travail « à Calgary ou à Mississauga ${ }^{4}$ ».

Le cœur économique et démographique du Canada est situé le long du corridor Windsor-Québec qui englobe Toronto et Montréal. Plus à l'est, les provinces atlantiques essaient de rénover des économies mises à mal par les crises ayant frappé les secteurs traditionnels, pêche, industries minières et forestières essentiellement. Les politiques d'ajustement et la recherche de solutions souvent originales de développement permettent à certaines régions du Canada atlantique de se rapprocher de la moyenne nationale d'un pays qui figure parmi les plus riches du monde. Tel n'est pas encore le cas de Terre-Neuve et Labrador, la plus orientale de ses provinces, et encore moins, à l'intérieur de celle-ci, des régions littorales du Sud (tableau 3). 
Tableau 3 : Données économiques comparées de Fortune (péninsule de Burin), de la province de Terre-Neuve et Labrador et du Canada

\begin{tabular}{|l|c|c|c|}
\hline & Fortune & $\begin{array}{c}\text { Terre-Neuve } \\
\text { et Labrador }\end{array}$ & Canada \\
\hline Taux de chômage & 58,3 & 25,1 & 10,1 \\
Revenu moyen annuel des personnes ayant déclaré un revenu (CAN \$) & 15392 & 19710 & 25117 \\
Revenu total moyen annuel des familles (CAN \$) & 31877 & 42992 & 53792 \\
Valeur moyenne des logements (CAN \$) & 41668 & 70835 & 147877 \\
\hline
\end{tabular}

(source : Statistiques Canada, 1996)

Saint-Pierre et Miquelon et la péninsule de Burin sont donc confrontés à un même mal, la dépendance, traité de façon radicalement différente par leur gouvernement respectif. Sortir de ces dépendances, ici aux subsides métropolitaines, là aux décisions drastiques peu accompagnées, constitue un défi commun aux voisins français et canadiens. Cependant, on peut considérer que, malgré la petite taille de l'archipel français, la péninsule de Burin constitue pour lui un cadre trop étroit de coopération et n'en fournit donc, malgré la proximité, qu'un élément marginal. Outre le fait que les populations concernées sont peu nombreuses, le talus économique conduit à la perpétuation de rapports marqués par des relations de type dominant-dominé. L'évocation des Terre-Neuviens arrivant les dimanches d'été par le traversier de Fortune pour passer la journée à Saint-Pierre est quelque peu teintée de commisération puisqu'on souligne fréquemment le manque de moyens financiers ne leur permettant pas d'y consommer. À l'opposé, un certain nombre d'habitants de Saint-Pierre et Miquelon profite du différentiel économique pour acquérir des maisons à Terre-Neuve. Sur la péninsule de Burin, environ 70 habitations, laissées vacantes par les migrations économiques, ont été achetées à un prix très accessible aux confortables revenus d'une partie de la population.

En matière de coopération avec la péninsule de Burin, la piste la plus fréquemment mentionnée concerne la captation d'une partie des touristes séjournant à Terre-Neuve. Cette stratégie s'appuie sur le constat que ce qui est bon pour Saint-Pierre et Miquelon est bon pour la péninsule de Burin puisque, pour se rendre par la voie terrestre sur l'archipel français, les touristes doivent la traverser. L'image associant la France et l'Amérique du Nord est mise en avant pour attirer les visiteurs. Dans l'esprit de ses promoteurs, la combinaison d'une part d'un paysage et d'un aspect urbain nord américain et d'autre part d'un certain nombre de signes extérieurs d'une culture française - la baguette de pain, la place Général-de-Gaulle, les gendarmes - serait de nature à attirer des touristes en mal d'exotisme (photo 1). La clientèle des États-Unis est expressément visée, mais même si les objectifs sont modestes, les handicaps structurels, notamment le coût d'accès, la cherté de la vie et l'inadéquation du parc hôtelier aux standards américains, constituent des obstacles difficilement surmontables d'autant plus que cette dualité ne saute pas spontanément aux yeux. 
Photo 1 : une rue de Saint-Pierre : paysage urbain nord américain et norme française représentée par le poteau électrique

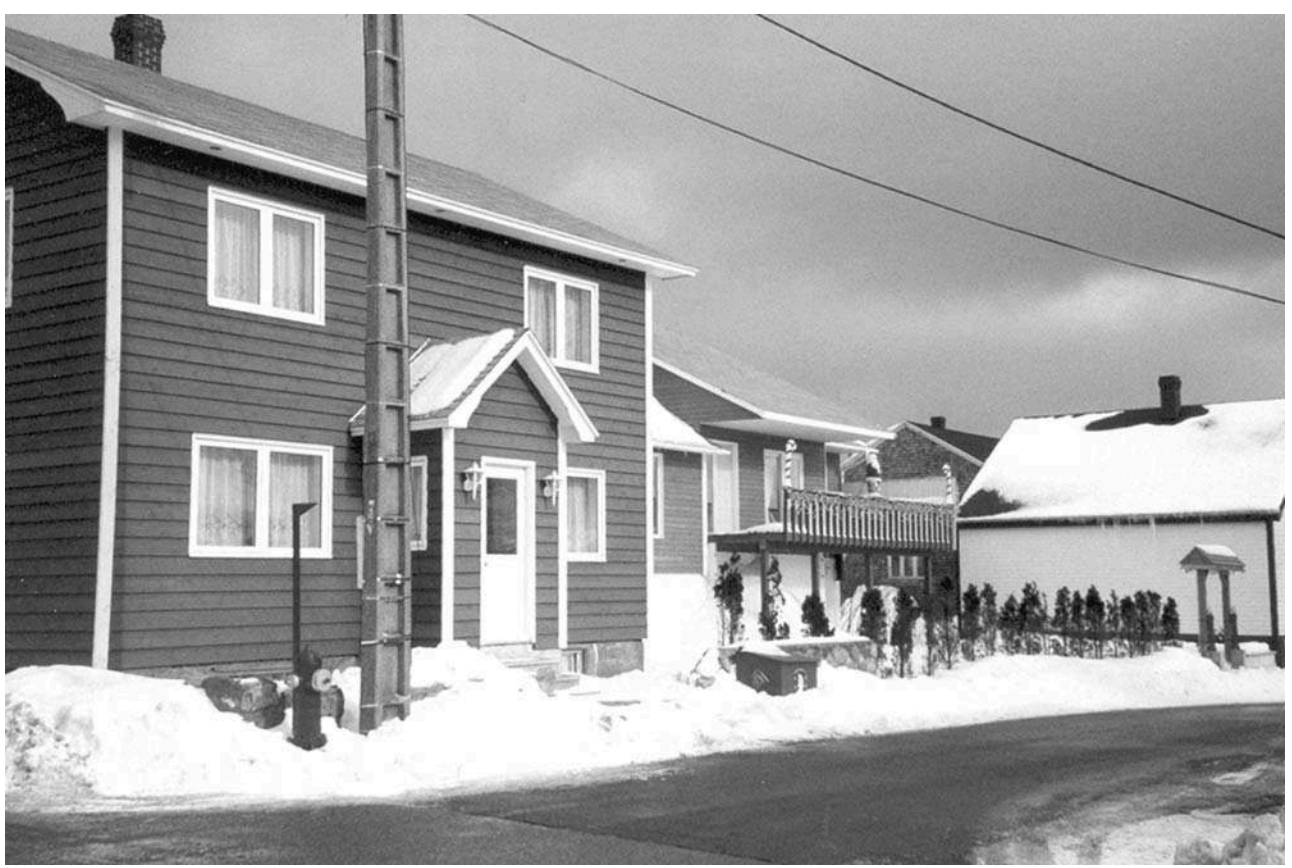

(C. Fleury)

Encore plus délicate à promouvoir, la référence pourtant mise en avant de "SaintPierre et Miquelon réserve d'espace naturel», ne peut pas constituer une spécificité dans un environnement nord américain dont le moins qu'on puisse dire est qu'il ne manque pas d'espaces forestiers, lacustres et littoraux préservés.

La coopération régionale entre voisins immédiats se construit donc sur une tonalité mineure, essentiellement en raison du talus économique séparant des communautés de faible importance numérique de part et d'autre de la frontière. C'est au-delà de son voisinage immédiat que Saint-Pierre et Miquelon peut prétendre élargir la palette de ses relations avec le Canada.

\section{Saint-Pierre et Miquelon et le Canada : un spectre élargi des relations frontalières}

Sur l'archipel, la question des relations avec le Canada est un sujet passionnel. Chacun a son avis, construction mentale élaborée en fonction de sa position sociale, de ses liens familiaux, de ses origines, de ses intérêts économiques, commerciaux, financiers. Certains ne croient qu'au développement des relations avec Terre-Neuve, d'autres ne jurent que par le rapprochement avec les communautés francophones du Canada atlantique, d'autres encore rejettent la coopération régionale en agitant le spectre de l'assimilation par le Canada. Certains enfin estiment que Saint-Pierre et Miquelon peut jouer de toute une gamme de particularités, culturelle, économique, institutionnelle, fiscale, douanière.

Au-delà de la péninsule de Burin, Saint-Jean, capitale de la province Terre-Neuve et Labrador, représente l'échappée la plus utilisée par les habitants de Saint-Pierre et Miquelon. Ce choix est d'abord celui de la proximité spatiale. Le coût d'un vol Saint- 
Pierre/Saint-Jean de $150 €$ peut être considéré comme accessible comparé à celui des liaisons vers Halifax $(300 €)$ et Montréal $(500 €)$. Or l'avion constitue la seule possibilité de sortir de l'archipel, hormis en été la possibilité d'emprunter le traversier vers Fortune qui est situé à 350 kilomètres de Saint-Jean. La capitale de la province de TerreNeuve et Labrador joue un rôle de poumon urbain, de nature à assouvir le besoin de consommation d'une population pouvant bénéficier d'un niveau de prix environ deux fois moins élevé que chez elle. Elle joue également un rôle fonctionnel particulièrement important pour les habitants de Saint-Pierre et Miquelon dans le domaine de la santé. Cette question est en effet cruciale puisque l'état de l'hôpital de Saint-Pierre, son niveau d'équipement et la palette des spécialités médicales qui y sont pratiquées ne peuvent garantir une sécurité sanitaire satisfaisante à la population. La Caisse de Prévoyance Sociale (CPS), organisme public gestionnaire des assurés sociaux a donc mis en place un dispositif de soins à l'extérieur de l'archipel. L'hôpital de Saint-Jean de Terre-Neuve en représente le principal élément. Les retombées pour l'économie terreneuvienne ne sont pas négligeables. Selon les responsables de la CPS, Environ $2 \mathrm{M} €$ sont annuellement versés à l'hôpital de Saint-Jean. Une somme équivalente est injectée dans l'économie de la province grâce aux dépenses annexes effectuées par les habitants de Saint-Pierre et Miquelon dans le cadre de leurs déplacements sanitaires.

Dans l'autre sens, la proximité joue également dans le cas de Francoforum, institut d'apprentissage de la langue française situé à Saint-Pierre. La clientèle de cet organisme est en quasi totalité issue de Terre-Neuve. Les tentatives d'élargir la prospection vers la Nouvelle-Écosse sont obérées par le facteur distance-coût qui conduit les éventuels stagiaires à plutôt se tourner vers les instituts situés au Québec ou au Nouveau-Brunswick.

Contrairement aux relations avec Terre-Neuve, province anglophone du Canada atlantique, qui s'établissent selon des critères économiques liés à la proximité spatiale, les relations avec les communautés francophones du Canada atlantique sont plutôt du registre d'une part de la proximité linguistique et d'autre part d'une référence pour la composante acadienne de l'archipel à une histoire commune, visant à légitimer une proximité culturelle.

Dans un contexte global de rappel des références identitaires, la réactivation des liens du passé entre les différentes ramifications d'un peuple acadien soumis à la dispersion est un phénomène notable. Il est mu par le dynamisme de la communauté acadienne du Nouveau-Brunswick. La Société Nationale Acadienne entretient d'étroites relations avec des organisations satellites, dont Saint-Pierre et Miquelon/Acadie, qui travaillent dans une optique de cohérence entre les actions identitaires, culturelles, éducatives, économiques. La pratique des commémorations est à ce titre révélateur. Dans l'esprit de leurs promoteurs, elles doivent servir de leviers à des coopérations éducatives, culturelles et in fine économiques. Ainsi en est-il de la préparation du $400^{\mathrm{e}}$ anniversaire de l'établissement des premiers colons français en Acadie et du $100^{\mathrm{e}}$ anniversaire de l'abandon du French Shore en 2004, et en 2005, du $350^{\mathrm{e}}$ anniversaire de l'épisode emblématique du Grand Dérangement. La commémoration, cérémonie ponctuelle dans l'espace et dans le temps, doit contribuer, au-delà du classique " devoir de mémoire » à la réactivation de liens qui sont d'éventuels vecteurs de projets. Une forme de « tourisme généalogique » tente dans ce même esprit de se mettre en place. Ainsi, «La fête aux Cormier » qui s'est déroulée au cours de l'été 2002 à Saint-Pierre a rassemblé les descendants d'une des souches acadiennes les plus prolifiques. 

forme concrète d'intégration existe déjà. Le gouvernement du Nouveau-Brunswick, seule province officiellement bilingue du Canada et foyer francophone le plus important du Canada atlantique, a en effet décidé d'appliquer les mêmes droits d'inscription et de scolarité aux étudiants venant de Saint-Pierre et Miquelon qu'à leurs propres ressortissants contrairement à Terre-Neuve où les étudiants de l'archipel doivent s'acquitter de droits deux fois plus élevés que leurs homologues canadiens. Dans le même esprit, les bourses accordées par le Conseil Général de Saint-Pierre et Miquelon ne sont plus exclusivement destinées à financer des études en métropole mais peuvent également concerner une scolarité à l'Université de Moncton ou dans un collège communautaire. Sur l'archipel, beaucoup d'appareils fonctionnent avec des normes canadiennes. La solution canadienne en matière d'études répond donc à des exigences de formation et de consommation.

Cependant en raison de la petite taille de l'archipel, les projets de coopération régionale ne peuvent que s'appuyer sur des actions nécessairement de faible envergure. Cette réalité pourrait évoluer si Saint-Pierre et Miquelon réussit, dans son environnement géographique nord américain, à jouer de ses particularités institutionnelles par rapport à la France et à l'Union européenne susceptible d'intéresser un certain nombre d'opérateurs canadiens. En effet, Saint-Pierre et Miquelon jouit d'une situation particulière par rapport à l'UE. Ses ressortissants comme ceux des autres PTOM français ont la pleine citoyenneté française. Ils sont électeurs et éligibles à l'Assemblée nationale, au Sénat et au Parlement européen. Ils sont porteurs du passeport européen et bénéficient en tant qu'individus des avantages y afférant lorsqu'ils sont sur le territoire communautaire. Mais le statut de Collectivité territoriale que l'archipel partage avec Mayotte lui permet de disposer au niveau français, et par extension, européen, de marges de manœuvre propres en matière fiscale et douanière. La perte de ces avantages était d'ailleurs l'argument principal de l'opposition locale quasi unanime à la mise en place de la départementalisation entre 1976 et 1985, dispositif destiné selon ses promoteurs métropolitains à adresser un signal fort au Canada en montrant une volonté d'ancrer plus encore l'archipel au sein de la République. Les avantages consentis aux PTOM par l'Union européenne dans les domaines commercial et douanier permettent à Saint-Pierre et Miquelon d'envisager de jouer de ses spécificités institutionnelles dans son contexte frontalier. Ils rappellent, en plus favorable puisqu'ils concernent des territoires liés à des États membres, les dispositions établies entre l'UE et les États de l'Afrique, de la Caraïbe et du Pacifique (ACP).

Des accords ont été renouvelés en décembre 2001, considérant l'archipel comme un pays tiers disposant d'une fiscalité autonome et bénéficiant d'une association avec l'UE permettant à ses marchandises y entrant d'être exonérées de droit de douane. Deux principes caractérisent le régime commercial adopté pour les produits originaires des PTOM. Il s'agit d'une part de l'accès total et illimité au marché communautaire, disposition qui n'implique aucun devoir de réciprocité, et d'autre part de l'abolition des droits de douane. Les principaux axes d'exploitation de ces avantages sont de deux ordres, d'une part les pratiques jouant sur les règles d'origine et d'autre part le système de transbordement.

Les Canadiens souhaitent s'ouvrir le marché européen. En effet, le Canada en qualité de pays tiers voit ses exportations vers l'Europe renchéries par les droits de douane. Les dispositions décrites ci-dessus intéressent les milieux industriels canadiens, disposés à 
instrumentaliser Saint-Pierre et Miquelon à travers son statut particulier afin d'en faire un " marchepied" "a gateway" pour reprendre deux termes entendus, vers l'Union européenne et son marché de plusieurs centaines de millions de personnes. Cependant le problème de l'origine des marchandises se pose. Pour qu'elles rentrent en Europe selon les conditions réservées aux produits originaires du PTOM, il faut une ouvraison, c'est-à-dire une transformation significative dont le niveau doit être déterminé de façon extrêmement précise pour chaque produit dans un objectif de conformité aux normes du pays duquel elles sont censées devenir originaires. Les dossiers que doivent monter les différents opérateurs en coopération avec le service des douanes garants du respect de la législation sont d'une grande complexité dans la mesure où il faut adapter chaque produit aux procédures d'ouvraison. Le champ d'exploitation de ces dispositions concerne essentiellement des produits alimentaires. Le projet concernant les homards est le plus avancé puisque le texte finalisant l'accord a été publié au Journal Officiel de l'Union européenne en septembre 2003. Saint-Pierre et Miquelon a obtenu une dérogation d'origine pour ce produit issu du Nouveau-Brunswick. Le transit par l'archipel de 225 tonnes par an exonère des $16 \%$ de droit de douane normalement appliqués aux produits étrangers à condition de le transformer, en l'occurrence sous forme de queues et pinces congelées.

Le transbordement est un autre axe de développement très particulier qui a déjà fonctionné pendant deux ans et demi. Il représente un bon exemple de l'exploitation des marginalités géographique et institutionnelle de Saint-Pierre et Miquelon. Son intérêt est lié à sa situation au débouché de la voie maritime du Saint-Laurent, à proximité de la grande route transatlantique reliant l'Amérique du Nord à l'Europe. Les plus importantes alumineries du Canada se situent au Québec, sur les rives des fleuves Saint-Laurent et Saguenay. Les bateaux transportant l'aluminium vers l'Europe passent à proximité de Saint-Pierre et Miquelon. Or, l'article 36 des accords UE/PTOM permet d'y dédouaner des produits destinés à l'Union européenne plutôt que de le faire dans un port européen. Toutes les formalités sont faites en passant. C'est Saint-Pierre et Miquelon qui perçoit les droits de douane dont les recettes lui sont déléguées, à deux conditions. Le trajet doit être direct et le tarif douanier pratiqué ne doit pas être inférieur à celui de l'Union. Le trafic d'aluminium n'est donc plus dédouané à Rotterdam. Saint-Pierre et Miquelon peut donc être considéré comme une station service avancée de l'UE en Amérique du Nord. La déclaration en douane concerne la valeur de la marchandise, le coût toujours très élevé de l'assurance et le coût du fret. Le dédouanement à Saint-Pierre et Miquelon permet de réduire les coûts du fret et de l'assurance puisque la distance entre le point de départ et l'entrée douanière en Europe est considérablement réduite. Les $6 \%$, montant des droits de douane sur une distance réduite, sont payés à Saint-Pierre et Miquelon. Autre avantage pour l'opérateur, le temps d'immobilisation du navire pour satisfaire à cette opération, environ 6 heures, est bien moins long dans la rade de Saint-Pierre que dans un grand port européen du Northern Range.

41 L'UE a interrompu cette pratique fin 1999 suite à une contestation de son service d'enquête (OLAF) à cause de l'existence d'une aide au transport sur les marchandises exportées de l'archipel. Le litige est à l'étude et les chances de reprise sont réelles. Cette pratique, qui a représenté un apport d'environ 3,5 millions d'euros par an pendant deux ans et demi, pourrait d'ailleurs s'appliquer à d'autres trafics passant à proximité de Saint-Pierre et Miquelon comme en témoigne le projet concernant une société canadienne qui fabrique des pièces d'avion pour Airbus. Dans le même ordre d'idée, on 
a envisagé de faire de Saint-Pierre et Miquelon et de ses services spatialement concentrés, un espace de compréhension des mécanismes administratifs, fiscaux, douaniers non seulement français mais surtout européens à destination des éventuels opérateurs canadiens ou plus largement nord américains.

Dans tout contexte frontalier, la dialectique entre proximités et altérités alimente l'ambivalence de relations marquées par des attractions et des répulsions. Dans le cas évoqué ici, la proximité spatiale avec Terre-Neuve induit toute une palette de comportements qui vont, selon les individus et les groupes sociaux, du rejet découlant du contentieux historique à la coopération mue par des intérêts économiques. Ainsi, la proximité culturelle avec les communautés francophones du Canada atlantique, associée au relatif éloignement géographique, atténue les dissensions liées aux stratégies géopolitiques et aux intérêts économiques divergents.

Il convient ici de prendre également en compte le fait que se rassemble de chaque côté de la ligne frontière une convergence d'altérités, spatiale, économique, institutionnelle. Nous sommes ainsi en présence de deux types d'insularité, celle du petit archipel coupé d'une métropole qui, au-delà des incompréhensions et du sentiment d'abandon, lui garantit la survie économique et celle d'une grande île où jouent peu de mécanismes d'ajustement à la crise commune de la pêche.

Le succès des stratégies alternatives de développement de Saint-Pierre et Miquelon dépend notamment de la capacité de l'archipel à se servir à des fins dynamiques de caractéristiques marginales souvent génératrices de dépendances. Les passerelles institutionnelles transfrontalières récemment mises en place et la nécessité d'appliquer un traitement spécifique aux dossiers concernant la mer vont dans ce sens, celui de l'intégration régionale.

Une approche classique et transversale considère les régions frontalières comme étant régies par un rapport de forces entre États voisins. L'exemple décrit ici montre l'importance des rapports verticaux de chaque côté de la ligne frontière. Ainsi, en ce qui concerne Saint-Pierre et Miquelon, les relations d'une part à l'intérieur de la communauté insulaire et d'autre part avec la métropole constituent des facteurs également déterminants. Associées aux transversalités frontalières aux différents niveaux des échelles spatiale et sociale, elles contribuent à l'étonnante diversité du spectre relationnel concernant une aussi petite communauté.

\section{BIBLIOGRAPHIE}

BRIÈRE (J.-F.), 1990. - La pêche française en Amérique du Nord au XVIII siècle, Québec,Fidès, 270 p.

Cermakian (J.), Metton (A.), Raveneau (J.), 1970. - « Saint-Pierre et Miquelon, les mutations d'une économie insulaire ", Annales de Géographie, vol. 79, n 436, p. 657-688.

GIRARDIN (Rodrigue), 1990. - Répertoire des Mariages célébrés à Saint-Pierre et Miquelon 1816-1889, Saint-Pierre, Imprimerie administrative, $135 \mathrm{p}$. 
GuYotjeannin (O.), 1986. - Saint-Pierre et Miquelon, Paris, L'Harmattan, 139 p.

HiLlER (J. K.), 1995. - « The 1904 Anglo-French Newfoundland Fisheries Convention: Another Look », Acadiensis, XXV, Fredericton, p. 82-98.

Institut d'Émission des Départements d'Outre-Mer, 2002. - Rapport annuel, Saint-Pierre et Miquelon, $97 \mathrm{p}$.

LABRECQUe (G.), 1998. - Les Frontières Maritimes Internationales, Montréal, Harmattan, 443 p.

RAFFESTIN (C.), 1983. - « Autour de la fonction sociale de la frontière », Espaces et Sociétés, $\mathrm{n}^{\circ}$ 70/71, Paris, p. 157-164.

SAVoIE (D. J.), 2000. - Le développement économique communautaire au Canada atlantique : illusion ou panacée, Université de Moncton, $130 \mathrm{p}$.

\section{NOTES}

1. Signalons cependant à ce sujet les travaux de Martijn C, (1996), "Les Mi'kmaks dans les registres paroissiaux de Saint-Pierre et Miquelon, 1763-1830», Recherches Amérindiennes au Québec, vol 26, n², p. 49-72.

2. Ce cas est à dissocier de celui fort répandu où une ZEE ne débouche pas sur la haute mer en raison de son contact avec une autre qui lui fait face. C'est le cas par exemple des ZEE française et anglaise dans la Manche.

3. Les DOM sont: Guadeloupe, Guyane, Martinique et Réunion. Les PTOM sont: Mayotte, Nouvelle-Calédonie, Polynésie, Saint-Pierre et Miquelon, Terres Australes et Antarctiques Françaises (TAAF), Wallis et Futuna.

4. Mississauga est un pôle industriel de l'agglomération de Toronto, première métropole économique du Canada.

\section{RÉSUMÉS}

Situé à 24 kilomètres de l'île canadienne de Terre-Neuve, l'archipel français de Saint-Pierre et Miquelon comptait environ 6300 habitants au recensement de 2001. Sa situation périphérique, à l'extrême est du continent nord américain, conduit à le considérer comme un espace insulaire marqué par la dépendance d'une part à la métropole et d'autre part à son voisin canadien. Dans ses relations avec celui-ci, deux axes sont envisagés, traités selon une double approche, diachronique et multiscalaire :

- La frontière maritime qui sépare la France et le Canada est l'objet de litiges qui mettent en évidence les difficultés d'adapter la course à la territorialisation de la mer par les États à des activités socio-économiques transfrontalières (pêche et exploitation d'hydrocarbures en l'occurrence);

- Toute frontière politique se caractérise par son ambivalence, caractérisée par un jeu d'ouvertures et de fermetures. Elle peut être considérée comme une zone d'échanges déterminés à la fois par la proximité spatiale et par les altérités politiques, institutionnelles, économiques, 
linguistiques, culturelles. Malgré sa petite taille et son faible poids démographique, Saint-Pierre et Miquelon présente à ce titre une grande diversité dans ses relations avec le Canada.

The French archipelago of Saint-Pierre and Miquelon lies $24 \mathrm{~km}$ off the Canadian Island of Newfoundland. The census of 2001 recorded 6.300 inhabitants. The fact that it is situated on a margin, at the eastern extremity of the North American continent, makes it an insular space marked by its dependence on the home country, on the one hand, and its Canadian neighbour, on the other. In its relationship with the latter, two axes are considered and are treated from two approaches, a historical one and a multiscalar one:

- the maritime border that separates France and Canada is a source of dispute which shows the difficulties of fitting the race to territorialize the sea by the States, to socioeconomic activities that cross borders (fishing and oil exploitation in this instance);

- any political border is characterized by its ambivalence, demonstrating a system of openings and closures. It can be considered as an area of exchanges which are determined by the spatial proximity as well as by the political, institutional, economical, linguistic and cultural differences. Despite its small size and the small number of inhabitants, Saint-Pierre and Miquelon, nevertheless, presents a great diversity in its relationships with Canada.

\section{INDEX}

Index géographique : France, Canada, Saint-Pierre-et-Miquelon, France d'outre-mer Keywords : border, development, fisheries, insularity, periphery, regional co-operation

Mots-clés : coopération régionale, développement, frontière, insularité, pêche, périphérie

\section{AUTEUR}

\section{CHRISTIAN FLEURY}

CRESO (Centre de Recherche sur les Espaces et les Sociétés) - Université de Caen, ESO, UMR 6590

- CNRS, fleury.cote@wanadoo.fr 